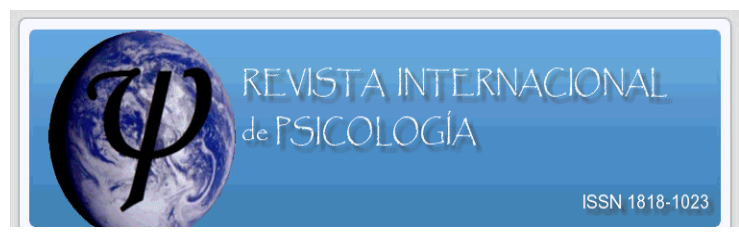

\title{
Predictores de respuestas psicológicas de duelo ante la pérdida de la salud
}

\section{Predictors of psychological responses of grief before the loss of health}

\author{
Dra. Melina Miaja Ávila \\ Dr. José Moral de la Rubia \\ Universidad Autónoma de Nuevo León
}

\begin{abstract}
Nota sobre los autores
No hay conflictos de intereses. Esta investigación fue realizada con el apoyo de la beca doctoral otorgada a la primera autora por el CONACYT.

Remita cualquier duda sobre este artículo al siguiente domicilio: Facultad de Psicología, Universidad Autónoma de Nuevo León. Calle Dr. Carlos Canseco 110. Col. Mitras Centro. Monterrey, NL, México. Teléfonos: (81) 83338233. Ext. 423. Fax. Ext. 103. Correos electrónicos:
\end{abstract}

Revisado por: Dra. María Guadalupe Ramírez Contreras Dr. Juan Pablo Sánchez Domínguez 


\section{Resumen}

Un concepto relevante para el estudio del duelo ante la pérdida de salud es la resiliencia, la cual contribuye en la asimilación y recuperación frente a la pérdida, y en un mayor bienestar psicológico y físico. La resiliencia no se ha aplicado dentro del marco teórico de fases de duelo de Kübler-Ross. Así cabría preguntarse: ¿cómo se relaciona la resiliencia en el proceso de duelo ante la pérdida de la salud?, y ¿cómo influyen la resiliencia sobre las respuestas psicológicas de duelo frente a otras variables significativas como religiosidad, ingresos económicos y escolaridad? Para responder estas interrogantes se estudió la relación de las respuestas psicológicas de duelo ante la pérdida de la salud con la resiliencia, variables sociodemográficas y de religiosidad; asimismo, se especificó y contrastó un modelo de ecuaciones estructurales para predecir las respuestas psicológicas de duelo. Se llevó a cabo un estudio descriptivo-correlacional con diseño no experimental transversal en una muestra no probabilística de 120 mujeres mexicanas en tratamiento oncológico. Se aplicaron dos instrumentos de medición: la escala de Respuestas Psicológicas de Duelo ante la Pérdida de la Salud (RPD-PS-38) y la Escala de Resiliencia Mexicana (RESI-M). Se encontró que la resiliencia tuvo una mayor asociación con las respuestas psicológicas de duelo que con las variables sociodemográficas y la religiosidad. El modelo predictivo tuvo un buen ajuste a los datos, el primer factor de segundo orden (menor reacción de afecto negativo ante la enfermedad) fue predicho por mayor apoyo familiar, mayor frecuencia de asistencia a los servicios religiosos y mayor ingreso económico familiar; el segundo factor de segundo orden (actitud positiva ante la enfermedad con apoyo en creencias religiosas) fue predicho por mayor competencia social, mayor asistencia a los servicios religiosos, menor escolaridad e ingreso económico familiar, siendo los dos factores de segundo orden del duelo independientes entre sí. Se concluye que la resiliencia es una variable importante en el proceso de duelo; como también lo es la religiosidad, que está asociada con escolaridad e ingresos bajos. Se 

en mujeres en tratamiento oncológico.

Palabras clave: resiliencia, duelo, cáncer, depresión, mujeres.

\begin{abstract}
Resilience is an important factor to consider when studying grief due to the loss of health. It not only helps the patient to be aware of his medical condition, but also it benefits the patient to become more psychologically and physically healthy. However, resilience has not been studied using Kübler-Ross' theoretical framework. Therefore, we formulated the following research questions: How resilience can help the patient dealing with grief which is originated from the loss of health? How does resilience influence a patient's grief response when other factors, such as spirituality, education, and economic status are taken into account? In order to answer these research questions, we studied the interaction between psychological grief responses of patients with regards to their resilience, socio-demographic variables, and spiritual background. A structural equation model was used to predict the psychological grief responses. This was carried out in a descriptive, correlational study using a cross-sectional non-experimental design, in a nonrandom sample of 120 Mexican women in cancer treatment. Two instruments were used: RDP-PS-38 and RESI-M. Our results showed that resilience was more closely associated to the psychological grief response than other variables, such as socio-demographics and spirituality. The predictive model had good fit. The second order's first factor (less negative reaction to the disease) was predicted by greater perceived family support, more frequent attendance at religious services, and higher family income. The second order's second factor (positive attitude towards the disease based on religious beliefs) was predicted by greater social skills, higher rate of attendance at religious services, lower level of education, and lower family income. These two grief second order's factors are independent from each other. We concluded that resilience is an
\end{abstract}


important variable in the grieving process, with spirituality being an equally important variable, especially among women with lower income and lower educational attainment. We suggest working on these previous aspects within group dynamics in order to facilitate the grieving process among women dealing with cancer treatment.

Key words: resilience, grief, cancer, depression, women.

\section{Introducción}

La resiliencia es un concepto nacido dentro del estudio del estrés postraumático que ha sido aplicado a diversas áreas de la psicología y la salud, entre ellas el proceso de duelo (Bonanno, 2005). Dentro de las teorías más importantes para conceptualizar el proceso de duelo está la de Kübler-Ross (2005) y en relación a la cual se han señalado factores de riesgo y protección, como variables sociodemográficas y de religiosidad (Maciejewski, Zhang, Block, \& Prigerson, 2007). El concepto de resiliencia no se ha aplicado dentro de este marco teórico ni se ha estimado su potencial predictivo frente a otros factores de riesgo y protección ya identificados, cuando podría ser útil para el campo de investigación y el desarrollo de estrategias de intervención.

\section{El duelo ante la pérdida de salud}

Etimológicamente el término duelo deriva de la palabra latina "duellum” que significa combate o lucha y se relaciona con la palabra latina "dolus" que significa dolor. El investigador mexicano Castro (2011) define duelo como la reacción dolorosa cuando uno se enfrenta o lucha contra la pérdida de algo significativo. En este mismo sentido, el investigador español Navarro (2006) señala que el duelo es una reacción principalmente emocional y comportamental en forma de sufrimiento y aflicción, cuando el vínculo afectivo se rompe. Así, el duelo es una reacción natural y necesaria ante la pérdida de un ser querido (familiar, amigo o mascota) o de una condición civil (divorcio), judicial (privación de la libertad), laboral (despido) o de salud 
(enfermedad degenerativa crónica), como la autora suizo-estadounidense Kübler-Ross (2005)

indica.

Todo individuo que se enfrenta a un proceso de enfermedad degenerativa crónica, como es el cáncer, experimenta un gran número de estresores, cambios y pérdidas (Kazak, 2005); asimismo, suele sufrir síntomas de ansiedad (Vespa, Ottaviani, Rosselli, Rossini, \& Balducci, 2013), depresión (Kersum \& Elia, 2007; Vespa et al., 2013) u otros síntomas adaptativos (Velázquez, García, Alvarado, \& Ángeles, 2007). Por este motivo, se tiende a asumir que el tener cáncer es una experiencia estresante, difícil y traumática.

Diversos autores consideran que los síntomas emocionales y conductuales evidenciados por las personas que padecen cáncer son reacciones adaptativas ante la enfermedad, y no trastornos psicopatológicos, ya que paulatinamente los pacientes se van acostumbrando a los cambios y pérdidas que van viviendo y con ello van disminuyendo las reacciones desadaptativas; es decir, las personas que pasan por esta situación de enfermedad atraviesan por un proceso de asimilación y acomodación, al cual se le conoce como proceso de duelo (Bonanno, 2005; Trask et al., 2003).

Uno de los modelos del proceso de duelo que ha tenido gran impacto en la tanatología y los cuidados paliativos es el de Kübler-Ross (2005). La autora señalaba que las personas que se encontraban en fase terminal, ante enfermedades degenerativas crónicas transitaban por cinco fases durante su proceso de duelo: negación, ira, negociación-pacto, depresión y aceptación.

La negación se refiere a la imposibilidad de reconocer como un hecho real que se padezca la enfermedad ("No creo estar enfermo"). La ira es definida como una reacción emocional de hostilidad, envidia y resentimiento por haber perdido la salud y es dirigida hacia todo el entorno, ya sea personal sanitario, familiares, amistades, incluso hacia Dios (“¿Por qué a mí?, es injusto tener está enfermedad"). El pacto/negociación se refiere a hacer promesas para alcanzar metas 
terapéuticas, de supervivencia o desarrollo personal ("Dios, prometo ser una mejor persona, si me ayudas a recuperar mi salud"). La depresión se refiere a sentimientos de un profundo vacío y dolor, adoptándose una actitud de claudicación ante la enfermedad ("Siento que no hay nada por lo que luchar"). La aceptación es definida como el reconocimiento de la enfermedad y situación de dolor y limitaciones que conlleva, sin buscar culpables, ni adoptar una posición de derrota, sino asumir una actitud responsable de lucha y supervivencia ("Acepto el hecho de tener la enfermedad y hago todo lo posible por adaptarme a los cambios que involucra la enfermedad") (Kübler-Ross, 2005).

El modelo de las cinco fases de duelo de Kübler-Ross (2005) ha sido contrastado empíricamente. Sólo el estudio de Maciejewski et al. (2007), cuestionado por su método de análisis, halló evidencia de la secuencia de fases. Por el contrario, los demás estudios no han encontrado apoyo empírico para la secuencia (Holland \& Neimeyer, 2010; Isla, Moncho, Gausch, \& Torras, 2008). Moral y Miaja (en prensa), al contrastar el modelo de Kübler-Ross y no hallar la secuencia de las cinco fases del duelo, propusieron que las fases fueran denominadas respuestas psicológicas de duelo ante la pérdida de salud y plantearon un modelo de seis factores correlacionados: negación, ira, pacto-negociación, fe-esperanza, depresión y aceptación. A estos 6 factores de primer orden subyacen dos dimensiones o factores de segundo orden independientes: reacción de afecto negativo (depresión e ira, así como negación y aceptación, pero con signo opuesto a los dos indicadores anteriores) y actitud positiva ante la enfermedad bajo el apoyo de creencias religiosas (pacto-negociación con un Ser Supremo y fe-esperanza, así como negación, pero con signo opuesto a los dos indicadores anteriores).

El marco teórico del presente artículo se asienta en Kübler-Ross para conceptualizar el duelo y la teoría de la personalidad sobre la resiliencia como factor protección para evitar complicaciones del duelo, aparte de variables muy trabajadas en el campo del abordaje del 
paciente crónico, como la religiosidad y las variables sociodemográficas de ingresos económicos

y escolaridad. Un marco teórico que podría integrar el planteamiento general del estudio sería el de la teoría transaccional o del estresor-afrontamiento/personalidad-respuesta de estrés (González, García, \& Landero, 2011). Según esta teoría, la respuesta de estrés que puede desencadenar un estímulo o situación está mediada por la evaluación que la persona hace de las demandas que requieren ser afrontadas y de los recursos con los que se cuenta para tal fin. El estrés resulta de una transacción entre la persona y el ambiente en la cual se valora que las demandas rebasan los recursos disponibles, y esta transacción está modulada por rasgos de personalidad que aportan o merman los recursos de afrontamiento (González et al., 2011). En el caso del planteamiento del presente estudio, el estresor sería estar en tratamiento oncológico; la resiliencia sería una variable de personalidad que modula la transacción entre la persona y ser tratada por cáncer, incrementando los recursos para afrontar las demandas percibidas; y la religiosidad, la escolaridad y el nivel socioeconómico serían recursos personales para el afrontamiento de la situación. La respuesta de estrés es conceptualizada desde el modelo de fases de duelo Kübler-Ross (2005), pero matizado como respuestas psicológicas coexistentes y no necesariamente secuenciales (Miaja \& Moral, 2014).

Se escogió el modelo de las etapas de duelo por su gran impacto en la tanatología presente y ser afín al marco de la teoría transaccional que posee gran alcance heurístico en la psicología de la salud. Ambas teorías contemplan a la persona como un agente que dota a su entorno de significados y se guía de una forma propositiva y planeada. Los modelos alternativos de corte humanista, gestáltico y psicoanalítico, también presentes en el área de la tanatología, poseen dificultades de instrumentalización y escaso bagaje empírico (Castro, 2011). No obstante, cabe señalarse que el duelo puede ser conceptualizado y abordado desde la teoría de los esquemas y 
sesgos cognitivos que es un marco teórico con amplio bagaje empírico, aunque poco considerado

dentro de la literatura de la tanatología (Valencia \& Dávila, 2010).

\section{La resiliencia como factor para un duelo exitoso ante la enfermedad}

Etimológicamente el término resiliencia procede de la palabra latina "resilio" que significa volver atrás, volver de un salto o rebotar. Con la connotación de salir y volver al estado anterior ante la adversidad fue aplicado a la psicología por Rutter a principios de la década de los noventa. Este investigador estadounidense define la resiliencia como la habilidad para surgir de la adversidad, adaptarse, recuperarse y acceder a una vida significativa y productiva (Rutter, 2007). Los investigadores chilenos Gómez y Kotliarenco (2010) definen la resiliencia como un proceso dinámico, constructivo, de origen interactivo, sociocultural que conduce a la optimización de los recursos humanos y permite sobreponerse a las situaciones adversas. En un sentido afín, los investigadores mexicanos González-Arratia, Valdez y González (2011) definen la resiliencia como un conjunto de atributos que posibilitan al individuo a superar la adversidad.

Diversos estudios señalan que la resiliencia se incrementa como resultado de superar una pérdida significativa o un acontecimiento potencialmente traumático, representando ésta una mayor capacidad de adaptación saludable (Bonanno, Westphal, \& Mancini, 2011; Mancini \& Bonanno, 2006; Roisman, 2005). Mancini y Bonanno (2006) encontraron que las personas frente a pérdidas significativas muestran emociones negativas, así como interrupciones menores y transitorias en su capacidad de funcionar, siendo estos fenómenos de menor intensidad en la medida en que las personas poseen mayor resiliencia.

Alcanzar la aceptación o acomodación ante la enfermedad es el final de un proceso de duelo exitoso (Kübler-Ross, 2005). Si la resiliencia se refiere a la capacidad de recuperación frente a la pérdida, la cual contribuye a un mayor bienestar psicológico y físico, a través de un afrontamiento más efectivo (Zautra, Hall, \& Murray, 2008), entonces es esperable que sea un 
factor protector de adaptación en el duelo ante la pérdida de la salud en pacientes con cáncer, dando lugar a mayor frecuencia de respuestas de aceptación y esperanza (Stewart \& Yuen, 2011), y menor frecuencia de respuestas de negación, ira y depresión (Min et al., 2013).

En este sentido, diversos estudios han encontrado que, en pacientes con enfermedades crónicas, la resiliencia se asocia con factores psicológicos, como son mayor aceptación (Bonanno et al., 2002), mayor esperanza, mayor locus de control interno, mayor optimismo y mayor apoyo social (Stewart \& Yuen, 2011), menor malestar emocional (Min et al., 2013), así como mayores estrategias de afrontamiento de reevaluación positiva, espiritualidad y afrontamiento activo (Stewart \& Yuen, 2011).

Dentro de la resiliencia se distinguen varios factores. Siguiendo a Palomar y Gómez (2010), se tendría la fortaleza del yo o confianza que posee la persona en sus propias capacidades, la competencia social, el apoyo familiar, el apoyo social y la estructura que la persona logra mantener en su vida cotidiana.

Considerando que la resiliencia como un factor protector para las respuestas negativas de duelo ante la pérdida de la salud no ha sido estudiada, cabe preguntarse ¿Cómo influye la resiliencia en el proceso de duelo ante la pérdida de la salud?

\section{La espiritualidad y religiosidad en el proceso de duelo ante la pérdida de la salud}

Otros aspectos que también se han considerado como factores protectores en el proceso de duelo ante pérdidas significativas son la espiritualidad y la religiosidad. Se puede definir espiritualidad como la tendencia a desarrollar los aspectos espirituales y transcendentes de la vida conforme algunas creencias religiosas que pueden ser personales o compartidas por una comunidad. Se puede definir religiosidad como la convicción en las creencias religiosas y observancia de los rituales y mandatos religiosos. Quinceno y Vinaccia (2009) señalan que la experiencia religiosa cobra mayor significado cuando se atraviesa por momentos difíciles y de 
incertidumbre. No obstante, las creencias y prácticas religiosas pueden tener efectos tanto positivos como negativos en el bienestar psicosocial de los pacientes con cáncer. Por un lado, pueden generar fortaleza y tranquilidad ante la adversidad, favorecer un mayor bienestar psicosocial y reducir el malestar emocional (Versano, 2011), pero, por otro lado, pueden favorecer el uso de un afrontamiento evitativo, depositando toda la responsabilidad en manos de un Ser Supremo (Klaassen, McDonald, \& James, 2006).

\section{Escolaridad e ingresos económicos en el proceso de duelo ante la pérdida de la salud}

Estatus socioeconómico alto y nivel de escolaridad alto se suelen señalar como factores protectores del duelo al contar la persona con mayores recursos psicológicos y materiales (Velázquez et al., 2007). Por el contrario, dificultades económicas y menores niveles de escolaridad conllevan mayor ansiedad y depresión ante la situación de enfermedad (OrnelasMejorada, Tufiño, \& Sánchez-Sosa, 2011), así como, menor aceptación de la enfermedad (Thompson et al., 2009). En este caso la escasez de recursos económicos genera la peor respuesta, lo que probablemente se evidencie más en países con desigualdades y desprotección en salud, como México (Aguilera, Marrufo, \& Montesinos, 2005); asimismo, la falta de recursos psicológicos para reconceptualizar y dar un significado positivo a la experiencia puede ser compensada por el apoyo en la fe y la religión. Versano (2011) halló que la relación entre calidad de vida y espiritualidad se veía incrementada en pacientes con bajo nivel educativo, esto es, que las personas con menos escolaridad en la medida que eran más espirituales llevaban mejor el proceso de duelo que las personas con más escolaridad fuesen o no espirituales. Este hallazgo, previamente, había sido reportado por Adler y Snibbe (2003). 


\section{Objetivos e hipótesis}

Considerando lo anterior, cabe preguntarse ¿Cuál es el efecto de la resiliencia sobre las respuestas psicológicas de duelo frente a otras variables significativas como religiosidad, ingresos económicos y escolaridad?

Desde las preguntas formuladas, la presente investigación enuncia como objetivos: 1) estudiar la relación de las respuestas psicológicas de duelo ante la pérdida de la salud con resiliencia, ingreso económico familiar, escolaridad y religiosidad (convicción en las creencias religiosas y frecuencia de asistencia a los servicios religiosos); y 2) generar modelos para predecir las respuestas psicológicas de duelo ante la pérdida de la salud con estas variables.

En relación con los objetivos enunciados se espera que una mayor resiliencia, mayor religiosidad, mayor ingreso económico familiar y mayor escolaridad predigan menor reacción de afecto negativo (Bonanno et al., 2002; Min et al., 2013; Stewart \& Yuen, 2011), siendo la resiliencia la variable con más peso predictivo (Zautra et al., 2008) y estando estos predictores correlacionados entre sí. La actitud positiva ante la enfermedad bajo creencias religiosas será predicha por mayor religiosidad, bajos ingresos económicos y baja escolaridad, e incluso más resiliencia dentro de la cultura mexicana. Así, la religiosidad puede jugar un papel muy importante para afrontar de una forma positiva el cáncer desde compromisos y pactos con Dios, especialmente en mujeres con bajos ingresos y baja escolaridad (Versano, 2011).

Considerando las dimensiones de las respuestas del duelo (Miaja y Moral, 2014) y las hipótesis formuladas, el esquema general del modelo queda representado en la Figura 1. 


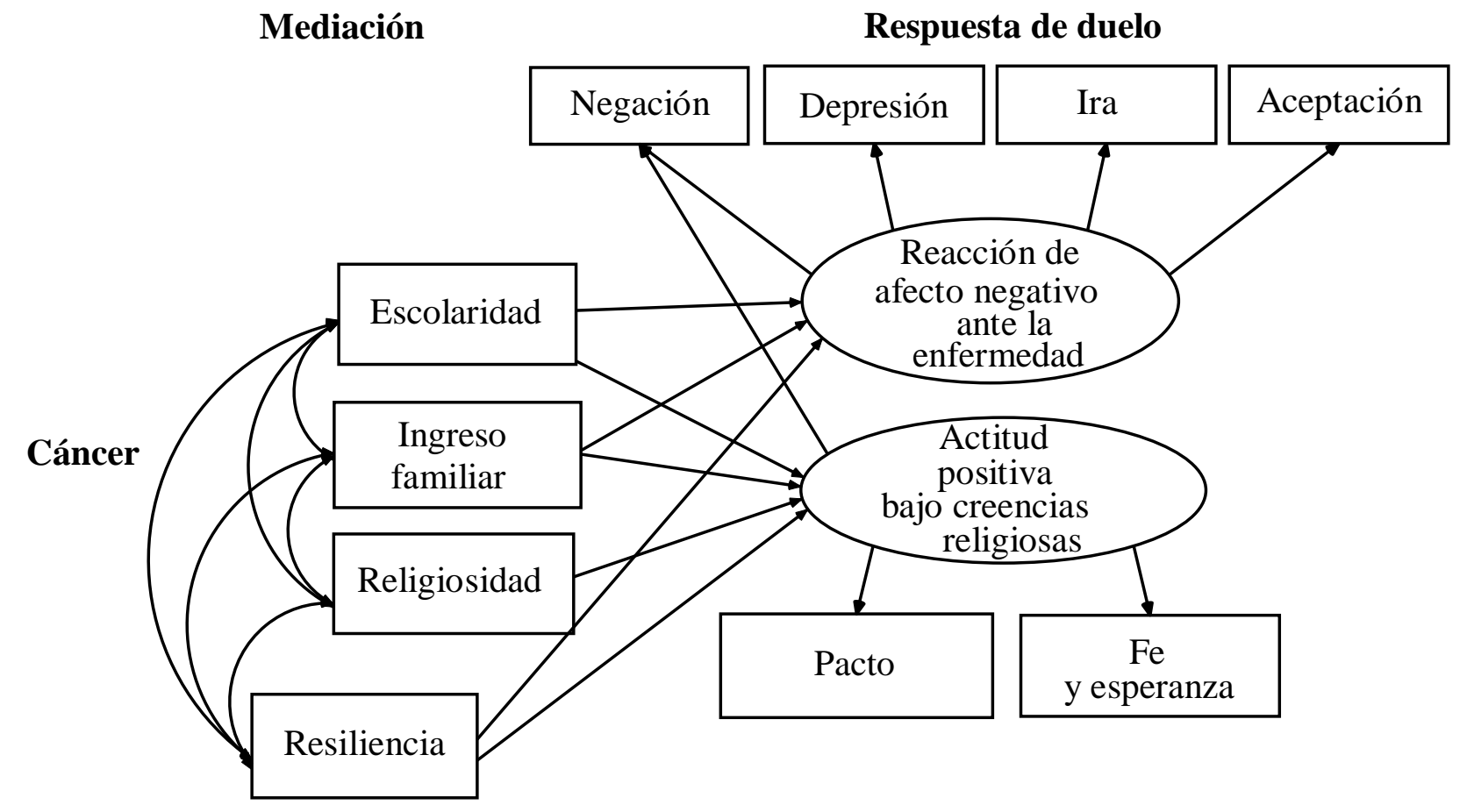

Figura 1. Modelo hipótetico. Dentro de la religiosidad puede considerar la convicción en la fe y frecuencia de práctica religiosa. Dentro de la resiliencia se puede considerar confianza en sí, competencia social, apoyo familiar, apoyo social y estructura de la vida cotidiana.

\section{Método}

\section{Diseño}

Se realizó un estudio de tipo correlacional con diseño no experimental transversal, usando un muestreo no probabilístico. Para estudiar los factores de riesgo y protección que predicen la respuesta de duelo ante el cáncer no se hizo viable y plantearía serios cuestionamientos éticos cualquier manipulación experimental de las variables, por eso se optó por un diseño ex post facto. Se midió el tiempo transcurrido desde el diagnóstico para aplicar un diseño transversal y evitar las dificultades operativas de un diseño longitudinal, considerando los permisos limitados concedidos a investigadores externos a las instituciones socio-sanitarias. Por este mismo motivo se optó por un muestreo no probabilístico de tipo incidental en lugar de probabilístico. 


\section{Participantes}

Los criterios de inclusión para formar parte de la muestra fueron: tener una enfermedad oncológica diagnosticada, recibir tratamiento oncológico, ser mayores de 15 años, saber leer y escribir para comprender adecuadamente las instrucciones y firmar el consentimiento informado. Los criterios de exclusión fueron: tener fatiga excesiva que dificultase la atención (a juicio de la encuestadora).

La muestra de tipo intencional quedó conformada por 120 mujeres. Se extrajo de siete lugares diferentes: 83 de las 120 pacientes (69\%) fueron entrevistadas en tres albergues (Cruz Rosa, A.B.P., Desafío del Noreste, A.C., y Posada del Peregrino de Caritas de Monterrey, A.B.P.), 31 (26\%) en dos clínicas (Clínica 0-19 y Opción Oncología), y 6 (5\%) en dos grupos de autoayuda (Asociación Mexicana Contra el Cáncer de Mama, A.C. de la Fundación CIM*ab y el grupo de autoayuda Mujeres con Cáncer).

La media de edad fue 51.84 años $(D E=13.63)$. La escolaridad varió de saber leer y escribir a estudios de posgrado terminados. La media de escolaridad correspondió a estudios de media superior sin terminar y el valor modal a primaria terminada (24\%). La media de ingresos económicos familiares al mes correspondió al intervalo de 6,000 a 11,999 pesos mexicanos.

Con respecto a la adscripción religiosa, 98 de las 120 mujeres (82\%) señalaron ser católicas, $15(12.5 \%)$ cristianas, $5(4.2 \%)$ pertenecer a otra religión distinta de la católica o cristiana y $2(1.7 \%)$ a ninguna religión. En relación con la convicción religiosa, 56 de las 120 participantes (46.7\%) dijeron creer totalmente, 39 (32.5\%) bastante, 19 (15.8\%) algo, 4 (3.3\%) poco y $2(1.7 \%)$ no creen en ninguna religión. En relación con la práctica religiosa, 66 de las 120 participantes (55\%) indicaron asistir con frecuencia a los eventos religiosos, 24 (20\%) con mucha frecuencia, $20(16.7 \%)$ en raras ocasiones a parte de eventos especiales y 10 (8.3\%) sólo a eventos especiales. 
En relación al tipo de cáncer, 60 de las 120 mujeres (50\%) presentaron cáncer de mama, $19(15.8 \%)$ cervicouterino, $14(11.7 \%)$ del sistema digestivo (colon, esófago y estómago), 9 (7.5\%) de huesos o articulaciones (sarcoma), 6 (5\%) de riñón, 4 (3.3\%) de glándulas endocrinas (ovarios y tiroides), 3 (2.5\%) linfoma, 2 (1.7\%) melanoma, 2 (1.7\%) leucemia y $1(0.8 \%)$ cerebral. La media del tiempo transcurrido desde el diagnóstico de cáncer fue de 1 año y 5 meses, $(D E=2.02)$. Con respecto al tratamiento oncológico, 58 de las 120 participantes $(48.3 \%)$ reportaron estar recibiendo radioterapia, 55 (45.8\%) quimioterapia, 5 (4.2\%) cirugía y 2 (1.7\%) hormonal.

\section{Instrumentos de medida}

Se aplicaron unas preguntas cerradas sobre datos sociodemográficos: sexo, edad, escolaridad, ingreso económico familiar mensual, adscripción religiosa, convicción en las creencias religiosas (“¿en qué grado se considera creyente?” con 4 opciones de respuestas: (de 1 = "poco" a 4 = "totalmente”) y frecuencia de asistencia a los servicios religiosos ("¿con qué frecuencia sigue los rituales o ceremonias?" con 4 opciones de respuestas: (de 1 = "solo en eventos especiales" a 4 = "con mucha frecuencia”).

La Escala de Resiliencia Mexicana (RESI-M) de Palomar y Gómez (2010) se desarrolló a partir de dos instrumentos que miden el constructo de resiliencia con la finalidad de adaptar y validar ambos instrumentos en población mexicana: Connor-Davidson Resilience Scale (CDRISC; Connor \& Davidson, 2003) y Resilience Scale for Adults (RSA; Friborg, Hjemdal, Rosenvinge, \& Martinussen, 2003). La escala RESI-M está integrada por 43 ítems tipo Likert con 4 opciones de respuestas: (de 1 = "totalmente en desacuerdo" a 4 = "totalmente de acuerdo"). La escala RESI-M tiene una estructura de 5 factores con valores de consistencia interna altos (de .79 a .92): fortaleza y confianza en sí mismo con 19 ítems ("creer en mí mismo me ayuda a superar los momentos difíciles"), competencia social con 8 ítems ("fácilmente me adapto a situaciones 
nuevas"), apoyo familiar con 6 ítems ("aún en momentos difíciles, nuestra familia tiene una actitud optimista hacia el futuro"), apoyo social con 5 ítems ("siempre tengo a alguien que puede ayudarme cuando lo necesito") y estructura con 5 ítems ("mantengo mi rutina aún en momentos dificiles"). Las puntuaciones de los 5 factores se obtienen por suma simple de ítems. A mayor puntuación mayor resiliencia.

La Escala de Respuestas Psicológicas de Duelo ante la Pérdida de la Salud (RPD-PS-38) de Miaja y Moral (2014) está integrada por 38 ítems tipo Likert con 5 opciones de respuestas: (de $1=$ "nunca" a 5 = "siempre"). La escala RPD-PS-38 tiene una estructura de 6 factores de primer orden correlacionados con valores de consistencia interna altos (de .72 a .85): negación con 7 ítems ("niego completamente que estoy enfermo"), ira con 6 ítems ("siento mucho coraje por tener la enfermedad"), promesas/negociación con un Ser Supremo con 7 ítems ("busco una alianza con un Ser Supremo para que me cure"), fe/esperanza con 4 ítems ("pienso que mi fe me ayudará a salir adelante"), depresión con 4 ítems ("no tengo ganas de hacer nada") y aceptación con 10 ítems ("estoy aprendiendo a vivir con la enfermedad"); y 2 factores de segundo orden o dimensiones independientes: reacción de afecto negativo y actitud positiva ante la enfermedad con apoyo en creencias religiosas. Las puntuaciones de los 6 factores de primer orden se obtienen por suma simple de ítems. La dimensión de segundo orden de reacción de afecto negativo se obtiene sumando directos los 17 ítems de depresión, ira y negación y sumando invertidos los 10 ítems de aceptación. La dimensión de segundo orden de actitud positiva se obtiene sumando directos los 11 ítems de promesas/negociación y fe/esperanza.

\section{Procedimiento}

El estudio siguió las especificaciones de manejo de pacientes de la Asociación Americana de Psicología (APA) (2002) y la Sociedad Mexicana de Psicología (SMP) (2007), así la 
investigación se realizó de manera competente y con el debido respeto a la dignidad y bienestar de las participantes (APA, std. 2.01; SMP, art. 49).

Antes de comenzar el estudio se proporcionó el protocolo de investigación a las siete instituciones anfitrionas con la finalidad de obtener las aprobaciones por las autoridades para llevar a cabo el proyecto de investigación. Se obtuvo la carta de consentimiento informado de los participantes, utilizando un lenguaje entendible y claro, informando la naturaleza de la investigación, haciéndoles saber sobre la confidencialidad del estudio y sobre el anonimato de los datos, así como el respetar la libertad de participar, declinar o retirarse de la investigación (APA, std., 8.02; SMP, art.118, 122, 124 y 132). En las pacientes menores de edad, la carta de consentimiento informado fue firmada por el cuidador primario y por la misma paciente.

Las participantes que aceptaron formar parte del estudio respondieron por escrito al instrumento en presencia de la psicóloga entrevistadora, en salas polivalentes, cuartos, pasillos o diversos lugares permitidos por cada una de las instituciones donde se llevó a cabo el estudio. El proyecto fue evaluado y aprobado en sus aspectos éticos por la comisión de posgrado de la Facultad de Psicología de la Universidad Autónoma de Nuevo León. La aplicación de los instrumentos tuvo una duración aproximada de 40 minutos. La muestra se recolectó de marzo a octubre del 2013.

\section{Análisis de datos}

En relación con el primer objetivo del estudio, las asociaciones de los factores de respuestas psicológicas de duelo con resiliencia, ingreso económico familiar, escolaridad y religiosidad se estimaron por medio del coeficiente de correlación producto-momento de Pearson. Se interpretó que valores de $r<.10$ reflejan una magnitud de asociación trivial, entre .10 y .29 pequeña, entre .30 y .49 mediana, entre .50 y .69 grande, entre .70 y .89 muy grande y $\geq .90$ unitaria (Ellis, 2010). 
En relación con el segundo objetivo, se calcularon dos modelos de regresión lineal múltiple para predecir los dos factores de segundo orden de duelo. A partir de estos dos modelos, se especificó y contrastó un modelo de ecuaciones estructurales lineales.

En los modelos de regresión lineal múltiple, se introdujeron como predictores aquellas variables que tuvieron correlación significativa con dos factores de segundo orden. El modelo se estimó por el procedimiento de pasos sucesivos (stepwise). El supuesto de independencia de los residuos se contrastó por el estadístico de Durbin-Watson; valores próximos a 2 reflejan independencia, entre 0 y 1 indican autocorrelación positiva de los residuos y entre 3 y 4 autocorrelación negativa (Chatterjee \& Simonoff, 2013). El supuesto de normalidad de la distribución de los residuos se contrastó por la prueba de Kolmogorov-Smirnov. La colinealidad entre los predictores del modelo se valoró desde los índices de tolerancia e inflación de la varianza; valores próximos a 1 indican baja colinealidad (Chatterjee \& Simonoff, 2013). El tamaño del efecto se calculó por el estadístico $f^{2}$ de Cohen $=\mathrm{R}^{2} /\left(1-\mathrm{R}^{2}\right)$. Se interpretó que valores del estadístico $f^{2}<.02$ reflejan un tamaño del efecto trivial, entre .02 y .14 pequeño, entre .15 y .34 mediano, y .35 a .99 grande $y \geq 1$ muy grande (Ellis, 2010).

La especificación del modelo estructural retoma el modelo de dos dimensiones de las respuestas de duelo desarrollado por Miaja y Moral (2014), al crear una escala RPD-PS-38 para evaluar las etapas de duelo de Kübler-Ross, las hipótesis formuladas en la introducción, así como los resultados de los modelos de regresión son calculados con los presentes datos.

Los parámetros e índices de ajuste del modelo estructural se calcularon por Máxima

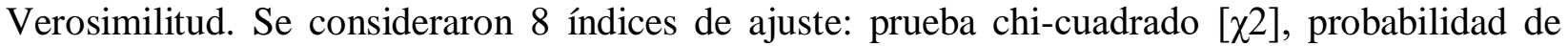
Bollen y Stine [ $p$ de B-S], residuo estandarizado cuadrático medio [RMS SR] de Jöreskog y Sörbom, residuo cuadrático medio de aproximación [RMSEA] de Steiger y Lind, índice de bondad de ajuste [GFI] y su modalidad corregida [AGFI] de Jöreskog y Sörbom, índice normado 
de ajuste $[\mathrm{NFI}]$ y no normado de ajuste $[\mathrm{NNFI}]$ de Bentler y Bonett. Se estipularon como valores de buen ajuste para los 9 índices: $p$ de $\chi^{2}$ y $p$ de $\mathrm{B}-\mathrm{S}>.05, \chi^{2} / \mathrm{gl} \leq 2$, RMSEA y RMS SR $\leq .05$, GFI $\geq .95$ y AGFI, NFI y NNFI $\geq .90 ;$ y como valores adecuados son: $p$ de $\chi 2$ y $p$ de B-S > .01, $\chi 2 / \mathrm{gl} \leq 3$, RMSEA y RMS SR $\leq .08, \mathrm{GFI} \geq .85$ y AGFI, NFI y NNFI $\geq .80$ (Kline, 2010). El cumplimiento del supuesto de normalidad multivariada se observó desde el valor estandarizado de la curtosis multivariada de Mardia. Al ser su valor mayor que 10, reflejando incumplimiento, se empleó el método de percentiles libres de sesgo para complementar el contraste de la significación de parámetros y la prueba de Bollen-Stine para complementar el contraste de la bondad de ajuste del modelo. La parsimonia del modelo se estimó desde la razón de parsimonia de James-Mulaik-Brett. Se estimaron los tamaños estandarizados de efecto directo sobre cada variable endógena $(\beta)$. Se interpretó que valores de $\beta<.10$ reflejan un tamaño de efecto trivial, entre .10 y .29 pequeño, entre .30 y .49 mediano, entre .50 y .69 grande, entre .70 y .89 muy grande $y \geq .90$ unitario (Kline, 2010).

\section{Resultados}

\section{Correlaciones de duelo con resiliencia, ingresos, escolaridad y religiosidad}

De las 48 correlaciones con resiliencia, 28 fueron estadísticamente significativas. La negación únicamente correlacionó negativamente con el apoyo familiar $(r=-.22, p=.02)$. La ira correlacionó negativamente con el puntaje total y todos sus factores, excepto con el factor de competencia social, con valores de -.20 a -.45. Pacto-negociación resultó independiente. Feesperanza correlacionó positivamente con el puntaje total, competencia social, apoyo familiar y estructura, con valores de .18 a .29. Depresión correlacionó negativamente con el puntaje total y los factores de fortaleza y confianza en uno mismo, y apoyo familiar, con valores de -.23 a -.31. Aceptación correlacionó positivamente con el puntaje total y todos sus factores, salvo el factor apoyo social, con valores de .33 a .47. Reacción de afecto negativo ante la enfermedad 
correlacionó negativamente con el puntaje total y todos los factores de resiliencia, con valores de -.23 a -.52. Actitud positiva ante la enfermedad correlacionó positivamente con el puntaje total y los factores de fortaleza y confianza en uno mismo, competencia social y estructura, con valores de .18 a .29 (véase Tabla 1 ).

De las 16 correlaciones con ingresos y escolaridad, 11 fueron estadísticamente significativas. Negación, pacto, fe-esperanza y depresión correlacionaron negativamente con ingreso y escolaridad, con valores de -.19 a -.31. Actitud positiva ante la enfermedad correlacionó negativamente con la escolaridad $(r=-.33, p<.01)$.

De las 16 correlaciones con religiosidad, 9 fueron estadísticamente significativas. Pacto ( $r$ $=.20, p=.03) \mathrm{y}$ actitud positiva ante la enfermedad $(r=.23, p=.01)$ correlacionaron positivamente con convicción en las creencias religiosas. Las correlaciones significativas de ira, depresión y reacción de afecto negativo con frecuencia de asistencia a los servicios religiosos fueron negativas, con valores de -.22 a -..30; y las correlaciones significativas de pacto, feesperanza, aceptación y actitud positiva fueron positivas, con valores de .19 a .34 (véase Tabla 1). 
Revista Internacional de Psicología www.revistapsicologia.org ISSN 1818-1023

Tabla 1

Instituto de la Familia Guatemala

Julio 2015

Correlaciones de las respuestas psicológicas del duelo con variables sociodemográficas y de resiliencia

Variables

$$
\text { Respuestas psicológicas de duelo }
$$

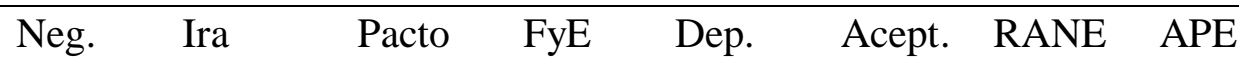

\begin{tabular}{|c|c|c|c|c|c|c|c|c|}
\hline Ing. & $-.31 * * *$ & $-.14 \mathrm{~ns}$ & $-.31 * * *$ & $-.26 * *$ & $-.22^{*}$ & $.11 \mathrm{~ns}$ & $-.22^{*}$ & $-.36 * * *$ \\
\hline Esc. & $-.23 *$ & $-.07 \mathrm{~ns}$ & $-.30 * * *$ & $-.29 * *$ & $-.19 *$ & $.11 \mathrm{~ns}$ & $-.16 \mathrm{~ns}$ & $-.33 * * *$ \\
\hline CCR & $.11 \mathrm{~ns}$ & $<-.01 \mathrm{~ns}$ & $.20^{*}$ & $.17 \mathrm{~ns}$ & $-.10 \mathrm{~ns}$ & $.08 \mathrm{~ns}$ & $-.07 \mathrm{~ns}$ & $.23^{*}$ \\
\hline FASR & $-.02 \mathrm{~ns}$ & $-.22 *$ & $.19 *$ & $.34 * * *$ & $-.23^{*}$ & $.19 *$ & $-.30 * * *$ & $.30 * * *$ \\
\hline RESI-M & $-.05 \mathrm{~ns}$ &.$- .33 * * *$ & $.10 \mathrm{~ns}$ & $.22 *$ & $-.25 * *$ & $.47 * * *$ & $-.44 * * *$ & $.22 *$ \\
\hline $\mathrm{F} \mathrm{y} \mathrm{C}$ & $-.04 \mathrm{~ns}$ & $-.26 * *$ & $.06 \mathrm{~ns}$ & $.16 \mathrm{~ns}$ & $-.23^{*}$ & $.47 * * *$ & $-.39 * * *$ & $.18^{*}$ \\
\hline CS & $.06 \mathrm{~ns}$ & $-.17 \mathrm{~ns}$ & $.09 \mathrm{~ns}$ & $.18^{*}$ & $-.15 \mathrm{~ns}$ & $.33 * * *$ & $-.24 * *$ & $.20 *$ \\
\hline $\mathrm{AF}$ & $-.22 *$ & $-.45 * * *$ & $.08 \mathrm{~ns}$ & $.20 *$ & $-.31 * * *$ & $.40 * * *$ & $-.52 * * *$ & $.13 \mathrm{~ns}$ \\
\hline AS & $-.06 \mathrm{~ns}$ & $-.20 *$ & $.03 \mathrm{~ns}$ & $.09 \mathrm{~ns}$ & $-.15 \mathrm{~ns}$ & $.17 \mathrm{~ns}$ & $-.23^{*}$ & $.05 \mathrm{~ns}$ \\
\hline E & $.03 \mathrm{~ns}$ & $-.29 * *$ & $.15 \mathrm{~ns}$ & $.29 * *$ & $-.15 n s$ & $.39 * *$ & $-.34 * *$ & $.29 * *$ \\
\hline
\end{tabular}

Significación de $r: \mathrm{ns}=p>.05, * p \leq .05, * * p \leq .01 \mathrm{y} * * * p \leq .001$. CCR $=$ convicción en las creencias religiosas. FASR $=$ Frecuencia de asistencia a los servicios religiosos. Respuestas psicológicas de duelo: FyE = Fe y esperanza. Acept. $=$ aceptación de la enfermedad. RESI-M = Puntaje total de la Escala de Resiliencia Mexicana, FyC = Fortaleza y confianza en sí mismo, $\mathrm{CS}=$ Competencia social, $\mathrm{AF}=$ Apoyo familiar, $\mathrm{AS}=$ Apoyo social y $\mathrm{E}=$ Estructura. RANE $=$ Reacción de afecto negativo ante la enfermedad, APE $=$ Actitud positiva ante la enfermedad.

\section{Predicción de la reacción de afecto negativo ante la enfermedad}

Se introdujeron inicialmente las 8 variables que presentaron correlación significativa: ingresos, frecuencia de asistencia a los servicios religiosos, el puntaje total de la escala de 
resiliencia (RESI-M), y sus factores de fortaleza y confianza en uno mismo, competencia social, apoyo familiar, apoyo social y estructura.

Al aplicar el método de pasos sucesivos, quedaron 3 variables en el modelo: apoyo familiar $(\beta=-.43)$, ingreso económico familiar $(\beta=-.24)$ y frecuencia de asistencia a los servicios religiosos $(\beta=-.17)$. El modelo explicó el $29 \%$ de la varianza de la reacción de afecto negativo, siendo su tamaño del efecto grande $\left(f^{2}\right.$ de Cohen $\left.=0.45\right)$. Debe señalarse que las tres variables predictoras no mostraron problemas de colinealidad, como indican los valores de tolerancia e inflación de la varianza próximos a uno (véase Tabla 2).

Tabla 2

Modelo de regresión para la reacción de afecto negativo ante la enfermedad

\begin{tabular}{llllllll}
\hline \multirow{2}{*}{ Modelo } & \multicolumn{2}{l}{ Coeficientes } & \multicolumn{2}{l}{ Significación } & \multicolumn{2}{l}{ Colinealidad } \\
\cline { 2 - 7 } & $B$ & $E E$ & $B$ & $t$ & $p$ & Tol & FIV \\
\hline Constante & 103.56 & 8.31 & & 12.46 & $<.01$ & \\
Apoyo familiar & -1.95 & 0.36 & -.43 & -5.42 & $<.01$ & .95 & 1.05 \\
Ingreso & -2.06 & 0.67 & -.24 & -3.09 & $<.01$ & .97 & 1.03 \\
FASR & -3.03 & 1.40 & -.17 & -2.16 & .03 & .95 & 1.05 \\
\hline
\end{tabular}

Método: Stepwise. Criterio: reacción de afecto negativo ante la enfermedad. $R=.56, R^{2}=.31, R^{2}$ aj. $=.29, E E E=$ 12.38. FASR $=$ Frecuencia de asistencia a los servicios religiosos.

El estadístico de Durbin-Watson fue 2.05, reflejando que no existe correlación serial entre los residuos, cumpliéndose así el supuesto de independencia de los residuos. La distribución de los residuos se ajustó a una curva normal $\left(Z_{K-S}=1.03, p=.24\right)$, cumpliéndose el supuesto de distribución normal de residuos. 


\section{Predicción de la actitud positiva ante la enfermedad}

Se introdujeron inicialmente las 8 variables que presentaron correlación significativa: ingresos, escolaridad, convicción religiosa, frecuencia de asistencia a los servicios religiosos, el puntaje total de la escala de resiliencia (RESI-M), y sus factores de fortaleza y confianza en uno mismo, competencia social y estructura.

Al aplicar el método de pasos sucesivos, quedaron 4 variables en el modelo: ingreso económico familiar $(\beta=-.24)$, frecuencia de asistencia a los servicios religiosos $(\beta=.17)$, competencia social $(\beta=.22)$ y escolaridad $(\beta=-.22)$. El modelo explica el $20 \%$ de la varianza de la actitud positiva, siendo el tamaño del efecto mediano $\left(f^{2}\right.$ de Cohen $\left.=0.28\right)$. Las dos variables sociodemográficas (ingresos y escolaridad) mostraron problemas de colinealidad, al no aproximarse a uno los valores de tolerancia e inflación (véase Tabla 3).

Tabla 3

Modelo de regresión para la actitud positiva ante la enfermedad

\begin{tabular}{llcccccc}
\hline \multirow{2}{*}{ Modelo } & \multicolumn{2}{l}{ Coeficientes } & \multicolumn{2}{l}{ Significación } & \multicolumn{2}{l}{ Colinealidad } \\
\cline { 2 - 7 } & $B$ & $E E$ & $\beta$ & $T$ & $p$ & Tol & $F I V$ \\
\hline Constante & 31.34 & 6.07 & & 5.16 & $<.01$ & & \\
Ingreso & -1.31 & 0.55 & -.24 & -2.38 & .02 & .66 & 1.52 \\
FASR & 1.91 & 0.96 & .17 & 1.99 & .04 & .94 & 1.06 \\
Competencia social & .53 & 0.21 & .22 & 2.56 & .01 & .91 & 1.10 \\
Escolaridad & -.74 & 0.36 & -.22 & -2.07 & .04 & .63 & 1.59 \\
& & & & & & & \\
\hline
\end{tabular}

Método: Stepwise. Criterio: actitud positiva ante la enfermedad. $R=.47, R^{2}=.22, R^{2}$ aj. $=.20$, EEE $=8.42$. FASR $=$ Frecuencia de asistencia a los servicios religiosos. 
El estadístico de Durbin-Watson fue 2.20, reflejando que no existe correlación serial entre los residuos, cumpliéndose así el supuesto de independencia de los residuos. La distribución de los residuos se ajustó a una curva normal $\left(Z_{K-S}=0.97, p=.30\right)$, cumpliéndose el supuesto de distribución normal de residuos.

\section{Modelo para predecir las respuestas psicológicas de duelo ante la pérdida de la salud}

Se especificó un modelo por ecuaciones estructurales lineales para predecir los dos factores de segundo orden de las respuestas psicológicas de duelo ante la enfermedad. El modelo de medida de reacción de afecto negativo quedó conformado por negación, ira, depresión y aceptación (con signo opuesto). El modelo de medida de actitud positiva ante la enfermedad quedó conformado por fe-esperanza y pacto-negociación. No se especificó ningún tipo de relación entre ambos factores al ser independientes. La reacción de afecto negativo fue predicha por ingreso económico familiar, frecuencia de asistencia a los servicios religiosos y apoyo familiar. Actitud positiva ante la enfermedad fue predicha por ingreso económico familiar, escolaridad, frecuencia de asistencia a los servicios religiosos y competencia social. Se especificaron dos correlaciones: entre ingreso económico familiar y escolaridad y entre apoyo familiar y competencia social (véase Figura 2).

La bondad de ajuste del modelo se mantuvo por la probabilidad de muestreo repetitivo de Bollen-Stine $(p$ de $\mathrm{B}-\mathrm{S}=774 / 2,000=.39)$ y la prueba de chi-cuadrado $(\chi 2[39, N=120]=50.04$, $p=.11)$. Los restantes índices reflejaron un ajuste de bueno $(\chi 2 / \mathrm{gl}=1.28, \mathrm{NNFI}=.94$ y RMS EA $=.05)$ a adecuado $(\mathrm{GFI}=.94, \mathrm{AGFI}=.89, \mathrm{NFI}=.84$ y RMS SR $=.08)$.

El modelo explicó el $49 \%$ de la varianza de reacción de afecto negativo ante la enfermedad y 57\% de la varianza de actitud positiva ante la enfermedad. Todos los parámetros fueron significativos por ambos métodos de estimación (ML y percentiles libres de sesgo). Desde la estimación ML, el tamaño de efecto del apoyo familiar sobre el factor de reacción de afecto 
negativo fue grande $(\beta=.59)$, el de la frecuencia de asistencia a servicios religiosos fue mediano ( $\beta=.31)$ y el del ingreso económico familiar fue pequeño $(\beta=.21)$. Los tamaños del efecto de la competencia social, del ingreso económico familiar y la escolaridad sobre el factor de actitud positiva ante la enfermedad fueron medianos ( $\beta=.40,-.35$ y -.31 , respectivamente), y el de frecuencia de asistencia a servicios religiosos fue pequeño $(\beta=.25)$ (véase Figura 2).

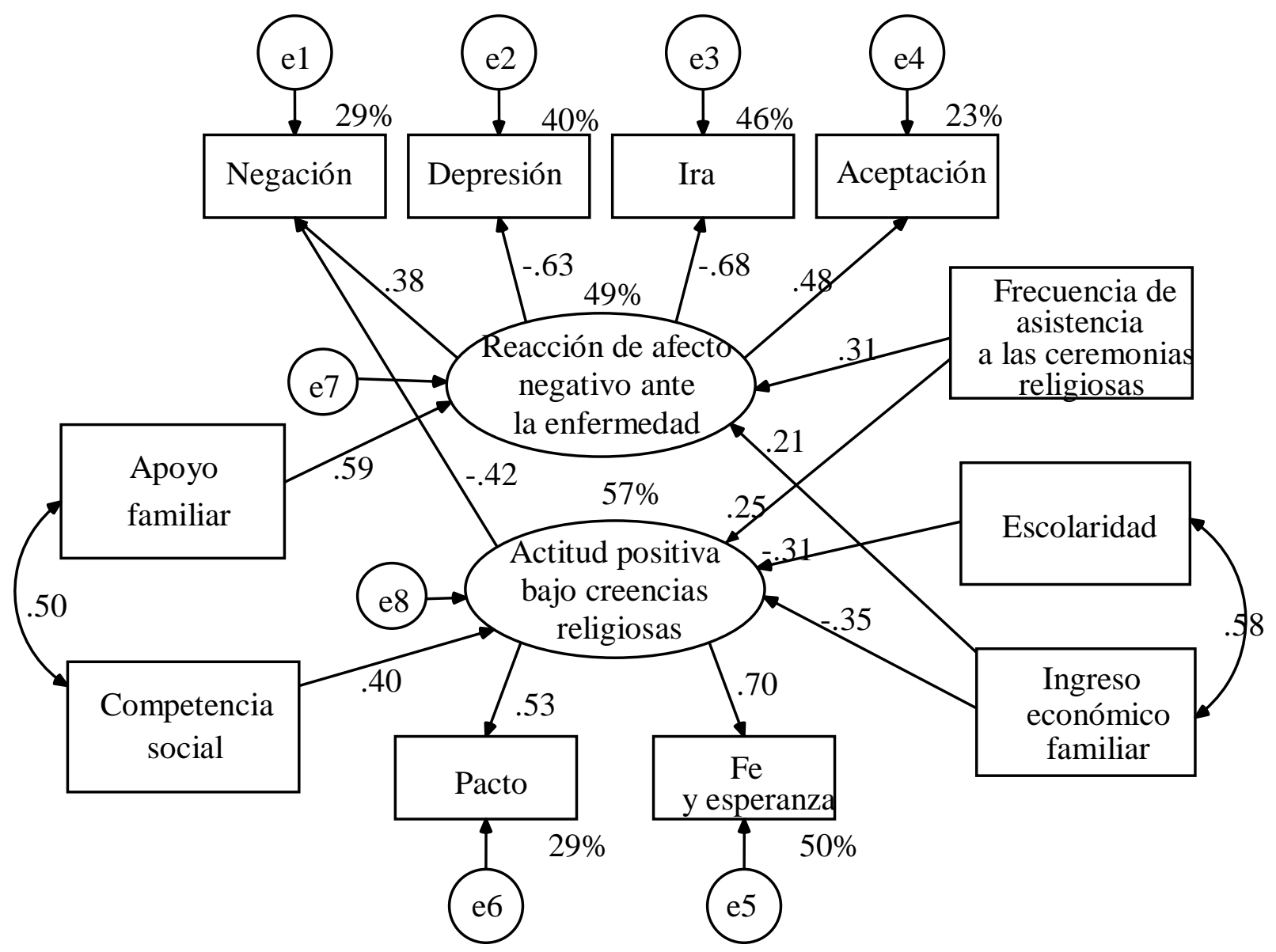

Figura 2. Modelo de regresión estructural con sus parámetros estandarizados estimados por Máxima Verosimilitud.

Debido al tamaño algo limitado de la muestra con una proporción de aproximadamente 5 participantes por cada parámetro a estimar $(\mathrm{N}: \mathrm{q}=120: 26)$ que corresponde al mínimo recomendado (Jackson, 2003), se calculó la potencia del contraste a partir del error de 

$=.049,90 \%$ IC: $0, .085)$ sea menor o igual que $.05(p=.492)$, se tomó como hipótesis nula el valor correspondiente al modelo independiente $\left(\mathrm{H}_{0}: \mathrm{RMSEA}=.200\right)$ y como hipótesis alternativa el valor del modelo especificado $\left(\mathrm{H}_{1}: \mathrm{RMSEA}=\right.$.049) (Preacher, Cai, \& MacCallum, 2007). Al ser los grados de libertad del modelo 39 y el tamaño de muestra de 120 participantes, se obtuvo una potencia unitaria estipulando una tasa de error de tipo $1 \mathrm{de} \alpha=.05$.

\section{Discusión}

Este estudio planteó como objetivos predecir las respuestas psicológicas del duelo con resiliencia y estimar el potencial predictivo de la resiliencia frente a otros predictores ya conocidos, como ingreso económico familiar, escolaridad y religiosidad, con la expectativa de que la resiliencia fuese un factor protector con un impacto mayor o semejante a la religiosidad. A continuación se discuten cada uno de estos predictores.

En relación con la resiliencia y conforme con las expectativas, los factores de negación, ira, depresión y la dimensión de reacción de afecto negativo ante la enfermedad correlacionaron de manera negativa; y los factores de fe-esperanza y aceptación y la dimensión de actitud positiva ante la enfermedad correlacionaron de manera positiva. Al igual que en el presente estudio, Bonanno et al. (2002) y Vinaccia, Quiceno y Remor (2012) observaron que la correlación de mayor magnitud de la resiliencia con el duelo aparece en la respuesta de aceptación de la enfermedad. Una mayor aceptación de sí mismo y de la vida favorece la comprensión de la enfermedad, así como una mayor salud mental y emocional. En este sentido, Coutu (2002) remarca que uno de los factores claves de la resiliencia es el aceptar la situación que se está viviendo. 
Conforme a los resultados de Bonanno et al. (2002) y las conclusiones de Stewart y Yuen (2011), entre los factores de la resiliencia, el apoyo familiar tuvo las correlaciones de mayor magnitud con las respuestas psicológicas de duelo ante la pérdida de la salud. Cuando se atraviesa por momentos de gran incertidumbre, el apoyo y la cohesión familiar aportan grandes beneficios a nivel físico y mental, como lo es una mejor recuperación (Cardoso, Ramos, Vaz, Rodríguez, \& Fernández, 2012).

En relación con las variables sociodemográficas, se encontró que a menores ingresos económicos y menor escolaridad aparece mayor negación, depresión y reacción de afecto negativo ante la enfermedad, lo que puede ser efecto de las dificultades que acarrea la incapacidad laboral y los costos del tratamiento. Precisamente, diversos estudios han encontrado que los problemas económicos y una menor escolaridad repercuten en la calidad de vida de los pacientes debido a que son una limitante en el acceso a la atención médica y seguimiento de los tratamientos (Martínez \& Guevel, 2013). Ornelas-Mejorada et al. (2011) hallaron que, en las personas con menores ingresos, las cuales usualmente tienen baja escolaridad, el costo de la incapacidad, atención médica y tratamientos genera ansiedad y depresión. No obstante, también bajos ingresos y baja escolaridad se asocian a pacto, fe-esperanza y actitud positiva ante la enfermedad, lo que parece contradictorio con el resultado previo. Esta contradicción desaparece cuando se entiende que estos tres factores hacen referencia al apoyo en creencias religiosas. Por lo tanto, las mujeres en situaciones más precarias y con menos recursos sufren más afecto negativo, por las penurias económicas que impone la enfermedad, y se apoyan más en la religión para afrontar dicha situación (Versano, 2011).

Se suele conceder a la religiosidad un mayor peso que a las variables sociodemográficas en el proceso de duelo (Klaassen et al., 2006). En el presente estudio destacó el ingreso económico como la variable de más peso tras los factores de resiliencia. Este resultado sí era 
y que se evidencian en los centros en los cuales se recogió la muestra. Tres albergues eran de beneficencia y dentro del rango de estratos socioeconómicos había personas con altos ingresos y que contaban con seguros privados de alta cobertura, los que garantizaban los procesos diagnósticos y terapéuticos necesarios y sin lista de espera.

En relación con la religiosidad, la convicción religiosa correlacionó positivamente con el pacto-negociación y actitud positiva ante la enfermedad. Estos resultados son afines con estudios previos en pacientes oncológicos, en los cuales la convicción religiosa juega un papel importante en el proceso de duelo, debido a que brinda un mayor sentido de paz y significado a la situación que están viviendo (McClain, Rosenfeld, \& Breitbart, 2003). Las prácticas religiosas suelen realizarse con mayor frecuencia ante momentos de pérdida, ayudando a las personas a afrontar los momentos difíciles por lo que están pasando (Klaassen, et al., 2006). En el presente estudio, se halló que una mayor frecuencia de asistencia a los servicios religiosos se relacionaba con menor ira, depresión y reacción de afecto negativo ante la enfermedad. Diversos estudios han encontrado resultados similares en donde las personas con una alta participación religiosa reportan menores síntomas depresivos (Kasen, Wickramaratne, Gameroff, \& Weissman, 2012; Pérez, Sandino, \& Gómez, 2005). Además, en el presente estudio, se encontró que a mayor frecuencia de asistencia a los servicios religiosos mayores son las puntuaciones en los factores de negociación-pacto, fe-esperanza, aceptación y actitud positiva ante la enfermedad. Quiceno y Vinaccia (2009) señalan que las prácticas religiosas favorecen el crecimiento espiritual continuo, propósito en la vida, y apoyo psicológico y social. Por lo tanto, en las mujeres del presente estudio, la convicción y las prácticas religiosas refuerzan la afectividad positiva durante el proceso de duelo ante la pérdida de la salud. Asimismo, Versano (2011) también, señala que la 
religiosidad y el encontrar un sentido a la experiencia de la enfermedad favorecían de manera significativa el bienestar psicosocial en pacientes con cáncer.

Desde este modelo predictivo integrador, aquellas personas que cuentan con mayor apoyo familiar (favorecido por mayor competencia social), con mayores ingresos económicos y que asisten con mayor frecuencia a los servicios religiosos reportan menor reacción de afecto negativo, caracterizada por menor ira y depresión y mayor negación-evitación y aceptación. Macias et al. (2004) encontraron unos resultados similares en el contexto de enfermedades mentales, destacando el apoyo de familiares y amigos entre los factores protectores de respuestas negativas de duelo.

Desde este modelo predictivo integrador, aquellas personas que tienen una mayor habilidad para interactuar con las personas de su entorno, que asisten con mayor frecuencia a los servicios religiosos y que cuentan con menores ingresos económicos y menor escolaridad logran mantener una actitud más positiva caracterizada por la confianza y pacto con un Ser supremo y la esperanza en la recuperación de la enfermedad, esto es, una actitud de lucha sostenida por creencias religiosas. Precisamente, México puede ser considerado un país con una religiosidad activa, en el que domina el culto guadalupano, y con mucha presencia de lo mágico-religioso en la vida cotidiana de sus ciudadanos, especialmente en aquéllos de clases sociales más desfavorecidas y menor escolaridad (Instituto Nacional de Estadística, Geografía e Informática, 2011); de ahí que estas mujeres en la situación de pérdida de la salud que sufren, por su propia carga cultural, fácilmente acudan a negociar con un Ser Supremo para buscar la recuperación de la salud, sobre todo las más desfavorecidas económica y socialmente.

Debe señalarse que actitud positiva ante la enfermedad con apoyo en creencias religiosas es independiente de la mayor o menor reacción de afecto negativo, pero está asociada a las variables sociodemográficas de ingreso y escolaridad. La actitud positiva con apoyo en la religión 
no motiva, ni mitiga el afecto negativo, sino que probablemente esté motivada por la cultura y la condición social desfavorecida. Además no debe confundirse esta dimensión del duelo con la religiosidad, ya que la convicción en las creencias religiosas sí está relacionada con la reacción de afecto negativo. Los más creyentes sufren menos afecto negativo. La dimensión de afecto positivo refleja como las mujeres de bajos recursos económicos y escolaridad se apoyan fundamentalmente en la religión ante una atención llena de demoras y limitaciones frente a las mujeres con mayores ingresos y escolaridad que cuentan con seguros privados de alta cobertura (Aguilera et al., 2005).

\section{Conclusiones}

En conclusión, la resiliencia es una variable importante en el proceso de duelo, como también lo son el ingreso económico, religiosidad y la escolaridad. Las magnitudes de asociación de las respuestas psicológicas de duelo son mayores con resiliencia que con las dos variables sociodemográficas y las dos variables de religiosidad. La magnitud de la asociación es mayor con ingreso económico que con escolaridad y es mayor con frecuencia de asistencia a los servicios religiosos que con convicción en las creencias religiosas. Con buen ajuste a los datos, una menor reacción de afecto negativo ante la enfermedad es predicha por mayor apoyo familiar, mayor frecuencia de asistencia a los servicios religiosos y mayor ingreso económico familiar, explicándose casi la mitad de su varianza. Una actitud más positiva ante la enfermedad (con apoyo en creencias religiosas) es predicha por mayor competencia social, mayor frecuencia de asistencia a los servicios religiosos, menor escolaridad e ingreso económico familiar, explicándose más de la mitad de su varianza. Ambas dimensiones del duelo son independientes entre sí. La actitud positiva ante la enfermedad con apoyo en creencias religiosas toma más peso entre mujeres de menores ingresos económicos y escolaridad. Se formula la hipótesis de que esté motivada por la cultura y la condición social desfavorecida. 
Como limitación del estudio debe mencionarse que el diseño fue ex post facto transversal, de ahí que sólo se habla de correlatos y predictores. Otra limitación fue que el estudio careció de una muestra poblacional, por lo que los resultados deben manejarse como hipótesis para futuras investigaciones en esta población y otras afines. Todos los datos fueron de autorreporte, por lo que se refieren a percepciones y evaluaciones hechas por las propias participantes y pueden diferir de datos procedentes de observaciones o pruebas proyectivas. Se podrían alegar problemas por incumplimiento de normalidad multivariada; no obstante, se acudió a los procedimientos de muestreo repetitivo con la extracción de un número grande de muestras, lo cual permite superar los problemas de ligera desviación de la normalidad multivariada, pero no de limitación de muestra (Kline, 2010; Nevitt \& Hancock, 2001). Aunque el tamaño de muestra fue algo limitado y próximo al mínimo recomendado de cinco participantes por cada parámetro a estimar (Jackson, 2003), se alcanzó una potencia unitaria desde el estadístico RMS EA en el contraste del modelo estructural y la mayoría de los efectos directos fueron altos sobre las variables endógenas, lo que disminuye este efecto en la estimación de parámetros y contraste del ajuste y da robustez al modelo (Preacher et al., 2007).

Para futuros estudios se sugiere incrementar el tamaño de la muestra y obtener una muestra equivalente de sexos, para estudiar la invarianza del modelo predictivo estructural de este estudio en hombres y mujeres mediante el contraste multigrupo. A su vez, se propone evaluar y potenciar el apoyo familiar y la competencia social para facilitar los procesos de duelo en mujeres en tratamiento oncológico. A tal fin se pueden emplear dinámicas de grupo. Por otra parte, es importante considerar y dar su lugar dentro del tratamiento de estas pacientes a sus creencias religiosas, los pactos y apoyos que entablan con Dios, los Santos y los espíritus de sus antepasados, especialmente en mujeres con bajos ingresos y baja escolaridad. Probablemente este 
espacio abierto a las creencias religiosas redunde en una mayor confianza en el equipo médico y

adherencia al tratamiento.

\section{Referencias}

Adler, N. E., \& Snibbe, A. C. (2003). The role of psychosocial processes in explaining the gradient between socioeconomic status and health. Current Directions in Psychological Science, 12(4), 119-123. doi:10.1111/1467-8721.01245

Aguilera, N., Marrufo, G. M., \& Montesinos, A. (2005). Desigualdad en salud en México: un análisis de sus determinantes. Ciudad de México: Universidad Iberoamericana.

American Psychological Association (2002). Ethical principles of psychologists and code of conduct. American Psychological Association, 57, 1060-1073. doi:10.1037/0003066X.57.12.1060

Bonanno, G. A. (2005). Resilience in the face of potential trauma. Current Directions in Psychological Science, 14(3), 135-138. doi:10.1111/j.0963-7214.2005.00347.x

Bonanno, G. A. Westphal, M., \& Mancini, A. D. (2011). Resilience to loss and potential trauma. Annual Review of Clinical Psychology, 7(1), 511-535. doi:10.1146/annurev-clinpsy032210-104526

Bonanno, G. A., Wortman, C. B., Lehman, D. R., Tweed, R. G., Haring, M., Sonnega, J., Carr, D., \& Nesse, R. M. (2002). Resilience to loss and chronic grief: A prospective study from preloss to 18-months postloss. Journal of Personality and Social Psychology, 83(5), 11501164. doi: 10.1037/0022-3514.83.5.1150

Cardoso, M. J., Ramos, M.I., Vaz, F. J., Rodríguez, L., \& Fernández, N. (2012). Influencia del apoyo familiar en momentos de gran incertidumbre. Revista Prisma Social, 8(1), 28-47.

Castro, M. (2011). Coaching tanatológico. Ciudad de México: Trillas. 
Chatterjee, S., \& Simonoff, J. (2013). Handbook of regression analysis (5th ed.). New York, NY: John Wiley \& Sons. doi:10.1002/9781118532843

Connor, K. M., \& Davidson, J. R. (2003). Development of a new resilience scale: the ConnorDavidson Resilience Scale (CD-RISC). Depression and Anxiety, 18(2), 76-82. doi:10.1002/da.10113

Coutu, D. L. (2002). How resilience works. Harvard Business Review, 80(5), 46-56.

Ellis, P. D. (2010). The essential guide to effect sizes: An introduction to statistical power, metaanalysis and the interpretation of research results. Cambridge, United Kingdom: Cambridge University Press.

Friborg, O., Hjemdal, O., Rosenvinge, J. H., \& Martinussen, M. (2003). A new rating scale for adult resilience: What are the central protective resources behind healthy adjustment? International Journal of Methods in Psychiatric Research, 12(1), 65-76. doi:10.1002/mpr.143

Gómez, E., \& Kotliarenco, M. A. (2010). Resiliencia Familiar: un enfoque de Investigación e intervención con familias multiproblemáticas. Revista de Psicología, 19(2), 103-131.

González, M. T., García, J., \& Landero, R. (2011). El papel de la teoría transaccional del estrés en el desarrollo de la fibromialgia: un modelo de ecuaciones estructurales. Actas Españolas de Psiquiatría, 39(2), 81-87.

González-Arratia, N. I., Valdez, J. L., \& González, S. (2011). Investigación en resiliencia: ¿que hemos aprendido? En J. Moral, J. L. Valdez \& N. I. González-Arratia (Eds.), Psicología y salud (pp. 157-172). Ciudad de México: Consorcio de Universidades de México.

Holland, J. M., \& Neimeyer, R. A. (2010). An examination of stage theory of grief among individuals bereaved by natural and violent causes: a meaning-oriented contribution. Journal of Death and Dying, 61(2), 103-120. doi:10.2190/OM.61.2.b 
Instituto Nacional de Estadística, Geografía e Informática (2011). Panorama de las religiones en México 2010. Aguascalientes, México: INEGI.

Isla, P., Moncho, J., Guasch, O., \& Torras, A. (2008). Proceso de adaptación a la diabetes mellitus tipo 1 (DM1). Concordancia con las etapas del proceso de duelo descrito por Kübler-Ross. Endocrinología y Nutrición, 55(2), 78-83.

Jackson, D. L. (2003). Revisiting sample size and number of parameter estimates: some support for the N:q hypothesis. Structural Equation Modeling: A Multidisciplinary Journal, 10(1), 128-141. doi:10.1207/S15328007SEM1001_6

Kasen, S., Wickramaratne, P., Gameroff, M. J., \& Weissman, M. M. (2012). Religiosity and resilience in persons at high risk for major depression. Psychology Medicine, 42(3), 509519. doi:10.1017/S0033291711001516

Kazak, A. E. (2005). Evidence based interventions for survivors of childhood cancer and their families. Journal of Pediatric Psychology, 30(1), 29-40. doi:10.1093/jpepsy/jsi013

Kersum, L. S., \& Elia, J. (2007). Depressive symptoms and SSRI use in pediatric oncology patients. Pediatric Blood \& Cancer, 49(7), 881-887. doi:10.1002/pbc.21208

Klaassen, D. W., McDonald, M. J., \& James, S. (2006). Advance in the study of religious and spiritual coping. En P. T. P. Wong \& L. C. J. Wong (Eds.), Handbook of multicultural perspectives on stress and coping (pp 105-132). New York: Springer. doi:10.1007/0-38726238-5_6

Kline, R. B. (2010). Principles and practice of structural equation modeling (3a. ed.). New York, NY: The Guilford Press.

Kübler-Ross, E. (2005). On grief and grieving: finding the meaning of grief through the five stages of loss. Nueva York, NY: Simon \& Schuster. 
Macias, C., Jones, D, Harvey, J., Barreira, P., Harding, C., \& Rodican, C. (2004). Bereavement in the context of serious mental illness. Psychiatric Service, 55(4), 421-426. doi:10.1176/appi.ps.55.4.421

Maciejewski, P. K., Zhang, B., Block, S. D., \& Prigerson, H. G. (2007). An empirical examination of the stage theory of grief. JAMA: Journal of the American Medical Association, 297(7), 716-724. doi:10.1001/jama.297.7.716

Mancini, A. D., \& Bonanno, G.A. (2006). Resilience in the face of potential trauma: Clinical practices and illustrations. Journal of Clinical Psychology, 62(8), 971-985. doi:10.1002/jclp.20283

Martínez, M. L., \& Guevel, C. G. (2013). Desigualdades sociales en la mortalidad por cáncer de cuello de útero en la Ciudad Autónoma de Buenos Aires, 1999-2003 y 2004-2006. Salud Colectiva, 9(2), 169-182.

McClain, C. S., Rosenfeld, B., \& Breitbart, W. (2003). Effect of spiritual well-being on end-oflife despair in terminally-ill cancer patients. The Lancet, 361, 1603-1607. doi:10.1016/S0140-6736(03)13310-7

Miaja, M., \& Moral, J. (2014). Desarrollo y validación de la Escala de Respuestas Psicológicas de Duelo ante la Pérdida de la Salud (RPD-PS-38). Psico-Oncología, 11(2-3) 369-387. doi:10.5209/rev_PSIC.2014.v11.n2-3.47395

Min, J. A., Yoon, S., Lee, C. U., Chae, J.H., Lee, C., Song, K. Y., \& Kim, T. S. (2013). Psychological resilience contributes to low emotional distress in cancer patients. Supportive Care in Cancer, 21(9), 2469-2476. doi:10.1007/s00520-013-1807-6

Moral, J., \& Miaja (en prensa). Contraste empírico del modelo de cinco fases de duelo de KüblerRoss en mujeres con cáncer. Pensamiento Psicológico, 13(1). 
Navarro, M. (2006). La muerte y el duelo como experiencia vital: acompañando el proceso de morir. Información Psicológica, 88, 12-21.

Nevitt, J., \& Hancock, G. R. (2001). Performance of bootstrapping approaches to model test statistics and parameter standard error estimation in structural equation modeling. Structural Equation Modeling, 8, 353-377. doi:10.1207/S15328007SEM0803_2

Ornelas-Mejorada, R. E., Tufiño, M. A., \& Sánchez-Sosa, J. J. (2011). Ansiedad y depresión en mujeres con cáncer de mama en radioterapia: Prevalencia y factores asociados. Acta de Investigación Psicológica, 1(3), 401-414.

Palomar, J., \& Gómez, N. E. (2010). Desarrollo de una escala de medición de la resiliencia con mexicanos (RESI-M). Interdisciplinaria, 27(1), 7-22.

Pérez, A., Sandino, C., \& Gómez, V. (2005). Relación entre depresión y práctica religiosa: un estudio exploratorio. Suma Psicológica, 12(1), 77-86.

Preacher, K. J., Cai, L., \& MacCallum, R. C. (2007). Alternatives to traditional model comparison strategies for covariance structure models. In T. D. Little, J. A. Bovaird, \& N. A. Card (Eds.), Modeling contextual effects in longitudinal studies (pp. 33-62). Mahwah, NJ: Lawrence Erlbaum Associates.

Quinceno, J. M., \& Vinaccia, S. (2009). La salud en el marco de la psicología de la religión y la espiritualidad. Diversitas, 5(2), 321-336.

Roisman, G. I. (2005). Conceptual clarifications in the study of resilience. American Psychologist, 60(3), 264-265. doi:10.1037/0003-066X.60.3.264

Rutter, M. (2007). Resilience, competence and coping. Child Abuse and Neglect, 31(3), 205-209. doi:10.1016/j.chiabu.2007.02.001

Sociedad Mexicana de Psicología (2007). Código ético del psicólogo (4ª ed.). Ciudad de México: Trillas. 
Stewart, D. E., \& Yuen, T. (2011). A systematic review of resilience in the physically ill. Psychosomatics, 52(3), 199-209. doi:10.1016/j.psym.2011.01.036

Thompson, G. N., Chochinov, H. M., Wilson, K. G., McPherson, C. J., Chary, S., O'Shea, F. M., Kuhl, D. R., Fainsinger, R. L., Gagnon, P. R. y Macmillan, K. A. (2009). Prognostic acceptance and the well-Being of patients receiving palliative care for cancer. Journal of Clinical Oncology, 27(34), 5757-5762. doi:10.1200/JCO.2009.22.9799

Trask, P. C., Paterson, A. G., Trask, C. L., Bares, C. B., Birt, J., \& Maan, C. (2003). Parent and adolescent adjustment to pediatric cancer: Associations with coping, social support, and family function. Journal of Pediatric Oncology Nursing, 20(1), 36-47. doi:10.1053/jpon.2003.5

Valencia, A. I., \& Dávila, Y. (2010). Intervención cognitivo-conductual para la elaboración del duelo en la diada madre-hijo. Uaricha Revista de Psicología, 14, 1-11.

Velázquez, A. G., García, M. A., Alvarado, S., \& Ángeles, J. A. (2007). Cáncer y psicología. Gamo, 6(3), 71-75.

Versano, S. (2011). Religion and spirituality as a coping mechanism with cancer. Bamberg: Universität Bamberg, Diss.

Vespa, A., Ottaviani, M., Rosselli, M., Rossini, S., \& Balducci, L. (2013). Evaluation of intrapsychic processes, anxiety, and depression in postmenopausal women affected by breast cancer: a case-control study. Supportive Care in Cancer, 21(5), 1281-1286. doi:10.1007/s00520-012-1659-5

Vinaccia, S., Quiceno, J. M., \& Remor, E. (2012). Resiliencia, percepción de enfermedad, creencias y afrontamiento espiritual-religioso en relación con la calidad de vida relacionada con la salud en enfermos crónicos colombianos. Anales de Psicología, 28(2), 366-377. doi:10.6018/analesps.28.2.148821 
Zautra, A. J., Hall, J. S., \& Murray, K. E. (2008). Resilience: a new integrative approach to health and mental health research. Health Psychology Review, 2(1), 41-64. doi:10.1080/17437190802298568 


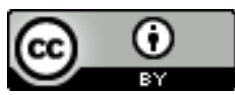

Este texto está protegido por una licencia Creative Commons $\underline{4.0}$.

Usted es libre para Compartir — copiar y redistribuir el material en cualquier medio o formato- y Adaptar el documen- to remezclar, transformar y crear a partir del material - para cualquier propósito, incluso comercialmente, siempre que cumpla la condición de:

Atribución: Usted debe reconocer el crédito de una obra de manera adecuada, proporcionar un enlace a la licencia, e in- dicar si se han realizado cambios. Puede hacerlo en cualquier forma razonable, pero no de forma tal que sugiera que tie- ne el apoyo del licenciante o lo recibe por el uso que hace.

$\underline{\text { Resumendelicencia }-\underline{\text { Textocompletodelalicencia }}}$ 